\title{
MODALITÉS NOUVELLES POUR LE STOCKAGE ET LE TRANSPORT DU LAIT EN VRAC
}

par

\author{
DIDIER-PETYT.
}

Ingénieur agricole E.N.A.G. Ingénieur conseil

\section{ORGANISATION DE RAMASSAGE DU LAIT EN FRANCE}

Le lait est collecté de ferme en ferme sur des camions à ridelles, de 2000 à $5000 \mathrm{~kg}$ de charge utile, transportant des bidons de 201 à 1001 de capacité, dirigés vers l'usine transformatrice, coopérative ou industrielle, dans un rayon de $70 \mathrm{~km}$ maximum, où lesdits bidons sont vidangés et lavés avant d'être réutilisés le lendemain. Le lait subissant des variations saisonnières du simple au double, en été notamment, où l'altération est plus rapide, la chaleur aidant, certains camions effectuent donc pendant six mois de l'année un double ramassage journalier.

Dans le prix de vente contrôlé du lait de consommation, les frais de ramassage admis sont de 3,90 AF par litre pour une masse totale de 28,90 AF comprenant tous les frais de traitement et de distribution, les frais de ramassage représentent donc 14 p. 100 des frais généraux; ce pourcentage doit être considéré comme une moyenne puisque dans certaines régions montagneuses il atteint 8,00 AF par litre; il y a lieu de noter que dans ce prix de $3,90 \mathrm{AF}$ sont compris les frais de réception à l'usine, contrôle, lavage de poteries qui sont de 1,5 AF par litre, augmenté de 1 AF s'il y avait réfrigération et stockage à l'usine.

Le volume de cette collecte destinée à la transformation fut, en 1963, de plus de cent soixante millions d'hectolitres, et, avec une conversion importante de crème fermière en lait, dans les régions de l'ouest notamment, cette collecte atteindra cette année deux cents millions d'hectolitres pour le million d'exploitations fermières existant en France.

Pour transporter une telle quantité, répartie sur 365 jours de l'année, car il n'existe pas de race laitière ne donnant pas de lait le dimanche! une flotte laitière estimée à plus de 20000 véhicules utilitaires est en service.

Pour diminuer ces frais de réception à l'usine, et notamment la manutention des bidons, de nombreuses entreprises laitières dans différentes régions, ramassent le lait dans des citernes, évitant ainsi, face à l'accroissement de la production, un achat de bidon- 
nages passé de mode ainsi que les frais d'extension des quais de réception et machines à laver supplémentaires.

Déjà, en 1947, j'avais réalisé un tel système à la suite de la pénurie de bidons résultant du passage des armées au débarquement, mais celui-ci a rencontré en Normandie notamment, des difficultés, car il laissait au producteur le soin du lavage des bidons, difficile à effectuer quand les vaches sont toute la journée à l'herbage.

Par ailleurs, il est démontré que le lait refroidi à la ferme dans les deux heures qui suivent la traite, peut se conserver sans s'altérer deux ou trois jours et doit être collecté dans des citernes routières de ramassage, doublant ainsi la densité kilométrique, travaillant à plein emploi, et permettant la fermeture dominicale des entreprises de traitement, ainsi que la concentration de ces entreprises dans des Unions Coopératives ou Industrielles.

Les économies réalisées sur les frais de ramassage, rattrapent et au-delà, les frais de refroidissement et de stockage à la ferme qui sont d'environ 2,50 AF par litre, représentant l'amortissement, l'énergie et l'entretien de ce matériel de stockage réfrigérant sur les lieux de production. Comme on peut ramasser le lait refroidi à n'importe quelle heure de la journée, matin et après-midi notamment, un seul camion peut effectuer 4 tournées en deux jours, supprimant ainsi trois chauffeurs et trois camions du système antérieur. En outre, il n'y a aucune perte de lait, car celui-ci est d'excellente qualité, et peut être transporté à longues distances, ce qui permet les concentrations et diminutions des frais du stade intermédiaire. Il est à remarquer, en effet, que le fonctionnement d'une tour de séchage à lait de 20000 l/heure coûte 15 AF de moins' au kilo que celui d'une tour de $3000 \mathrm{l} / \mathrm{h}$ eure.

Ce mode de ramassage s'est généralisé en Amérique, en Angleterre et en Nouvelle-Zélande depuis 1959. A titre indicatif, l'United Steel a fabriqué l'année dernière, uniquement pour le marché américain, 125000 tanks de réfrigération à la ferme et 8000 citernes de transport.

Le Génie Rural en France estime que le marché est ouvert pour 500000 appareils de réfrigération et 5000 camions citernes de ramassage et de transport à réaliser d'ici dix ans.

Déjà des zones pilotes ont été créées dans certaines zones laitières et de nombreuses firmes et chaudronneries se sont intéressées à la réalisation de ce matériel déjà en fonctionnement dans de nombreuses fermes.

On peut estimer qu'il y a environ 4 p. 100 de lait refroidi en France et fin de cette année environ 500 citernes de ramassage et 2500 tanks de réfrigération en place. 


\section{I. - Les équipements modernes de ramassage et de stockage du lait}

\section{a) Les citernes de ramassage.}

Trois équipements sont en présence :

- La citerne est mise sous vide par un compresseur actionné par la prise de force du camion et l'aspiration se fait directement par versage des pots dans un bac placé à l'arrière ou sur le côté du camion, ou une canne suceuse, employée de préférence pour le soutirage des tanks de ferme.

- L'aspiration est réalisée par une pompe actionnée soit directement sur la prise de force soit par transmission hydraulique.

- Les chassis reçoivent deux citernes elliptiques de faible hauteur, avec trou d'homme à hauteur d'homme, permettant le versage à bout de bras des pots à lait.

Les capacités varient entre 2000 et 6000 litres, mais déjà pour des laits refroidis et ramassés toutes les 48 heures, des capacités de 9000 à 15000 litres seront bientôt en service.

Ce matériel est réalisé en aluminium, acier inoxydable, ou plastique alimentaire.

b) Les installations frigorifiques pour le stockage du lait en vrac et en bidons.

Nous nous trouvons en présence de trois systèmes :

- Appareil à accumulation de froid, composé essentiellement d'une marmite norvégienne dans laquelle vient se loger la cuve à lait. L'évaporateur est plongé dans de l'eau qui se transforme partiellement en glace, dont la fusion, opérée généralement par une pompe auxiliaire, transmet ces frigories au lait dans lequel plonge un agitateur, facilitant l'absorption des frigories et empêchant la montée de crème. C'est ce type d'appareil qui est préconisé en France pour les petites capacités, 1501 maximum, dont le tank peut être amené sur chariot en bord de route. L'Office du Lait Anglais impose même ce système en raison des garanties pour le stockage qu'il apporte en cas de coupure de courant notamment.

- Appareil à détente directe, composé d'une enceinte et de la cuve à lait, dans laquelle est logé l'évaporateur. C'est le système d'agitation du lait qui permet le refroidissement. Ce type d'appareil 
qui n'offre pas les garanties précédentes, semblerait cependant consommer moins d'énergie quoique ayant un compresseur beaucoup plus important, ce qui entraîne un prix d'achat plus élevé.

- Le stockage du lait en bidons se fait par circulation d'eau glacée par accumulation et est préconisé en ramassage 48 heures pour les producteurs fournissant de 1 à 2 bidons seulement.

- Les appareils de plus grande capacité, 1000 litres et audessus, sont construits pour des exploitations importantes ou collectives à accumulation ou en détente directe, et sous vide en liaison avec la machine à traire pour éviter toute pollution atmosphérique préjudiciable surtout quand il y a stockage de plus de 48 heures

\section{1. - Isolation des citernes et des tanks réfrigérants}

Les citernes de ramassage n'ont pas besoin d'être isolées car il est démontré qu'en trois heures, temps normal d'une rotation, et par les plus fortes chaleurs, l'écart n'est pas de plus de trois degrés. Par contre, une isolation est nécessaire si le transport est effectué à longue distance. Cette isolation peut être effectuée en laine de verre, liège expansé ou polyurhétane en double parois, ou par un enduit vinylique projeté sur la paroi. Les citernes peuvent aussi être capotées avec une bâche doublée ouatinée en nylon.

Pour les tanks de stockage la paroi extérieure doit présenter une isolation thermique et la solution idéale serait un sandwich composé de deux viroles minces en acier inoxydable, avec injection à l'intérieur de polyurhétane à cellules fermées, évitant ainsi les phénomènes électrolytiques qui s'établissent avec le cuivre, le zinc ou le fer et mettent rapidement l'appareil hors d'état de marche.

\section{III. - L'acier inoxydable, matériau indispensable}

Dans le monde entier (Europe, Amérique, Australie, Indes, etc.) il existe des normes officielles qui spécifient pour la construction de tous les équipements en contact avec le lait, l'acier inoxydable à l'exclusion de tout autre matériau. Une telle obligation n'existe pas en France et peut être à la faveur de ce nouveau matériel à mettre en œuvre, pourrait elle être étudiée.

L'acier inoxydable possède en effet un certain nombre de qualités essentielles, du point de vue sanitaire, économique et esthétique. 
$1^{\circ}$ Amélioration de la qualité du lait par élimination efficace des bactéries.

Après vidange des cuves et citernes, il doit être procédé rapidement au nettoyage complet des surfaces. Du point de vue sanitaire, le critère le plus important auquel doit répondre un matériel en contact avec le lait ou tout autre substance alimentaire, est la possibilité d'élimination des bactéries par un nettoyage rapide et efficace.

Seul l'acier inoxydable permet une stérilisation effective. Ceci est attesté par les nombreux essais de comptage bactériens après lavage de différents matériaux (inox, plastique, aluminium) à l'aide de solution de produits détergents ou acide. D'ailleurs, certains matériaux autre que l'acier inoxydable se corroderaient au contact de solutions diluées d'acide nitrique ou d'hypochlorite, tandis que l'action des détergents y laisserait subsister un pourcentage important de bactéries, notamment le "Micrococcus aureus".

Dans une étude publiée dans l'American Journal of Public Health, vol. 43, no 2, février 1953, MM. G. M. Ridenour et E. H. Armbuster, professeurs à l'Université de Michigan (U.S.A.) décrivent les essais qu'ils ont effectués sur l'acier inoxydable, les plastiques, l'aluminium et le verre, par la méthode de comptage aux radioisotopes.

Les conclusions de cette étude sont les suivantes :

a) 97 p. 100 des bactéries « Micrococcus aureus» (les plus difficiles à éliminer) sont enlevées de l'acier inoxydable après un lavage à l'eau à $70^{\circ} \mathrm{C}$ contenant 0,25 p. 100 de détergent ordinaire suivi d'un rinçage à l'eau à la même température. Ce pourcentage de 97 p. 100 reste invariable quel que soit l'état de surface de l'acier inoxydable (neuf et usagé);

b) En ce qui concerne les plastiques et l'aluminium à l'état neuf, les pourcentages de bactéries éliminées dans les mêmes conditions varient de 56 à 91 p. 100. Ces résultats sont encore inférieurs quand les surfaces sont usagées. Cette constatation est logique puisque des matériaux tels que la porcelaine et les matières plastiques deviennent plus poreux une fois leur surface abîmée. Il n'en est pas de même pour l'acier inoxydable (voir $a$ ), métal parfaitement homogène et dur.

c) Les graphiques (voir fig. 1 et 2 en annexe) illustrent les résultats obtenus après cinq lavages et rinçages effectués dans des conditions identiques. La supériorité de l'acier inoxydable sur les autres matériaux, quel que soit le degré d'usure, est tout à fait évidente. 
Ces conclusions sont confirmées par différents travaux, tels que ceux de J. G. Davis The cleanability of various materials, extrait de The medical officer, 8 novembre $1962(110,299,302)$ et ceux de E. B. Masurovsky et W. K. Jordan (J. Dairy Sci., $41,1342,1958)$.

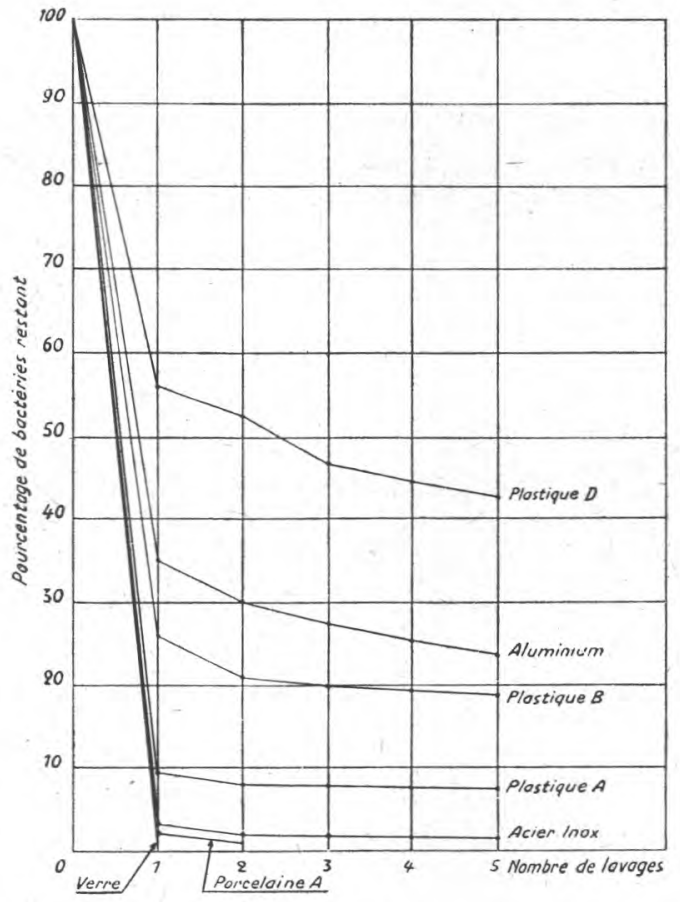

Figure 1

Pourcentage de M. aureus subsistant sur diverses surfaces non endommagées après un certain nombre de lavages avec une solution détergente suivie de rinçages à $70^{\circ} \mathrm{C}$.

D'une façon générale, une cuve destinée à contenir du lait peut s'endommager si un nettoyage adéquat n'est pas effectué rapidement après la vidange. En effet, des résidus de lait séché pourraient s'accrocher avec formation d'acide lactique et croissance bactérienne. Pour le nettoyage et la stérilisation, on peut utiliser soit des détergents en solution dans l'eau (à $70^{\circ} \mathrm{C}$ maximum), soit une solution d'acide nitrique diluée à 10 p. 100 (même température) ou encore une solution d'hypochlorite combiné au phosphate trisodique. 
L'acide nitrique dilué permet une stérilisation très efficace et bon marché. Quel que soit le traitement choisi, il faut qu'il soit suivi rapidement d'un rinçage soigné à l'eau chaude $\left(70^{\circ} \mathrm{C}\right)$. On conseille d'éviter d'utiliser des eaux dures à haute température, qui pourraient laisser des résidus calcaires sur les surfaces.

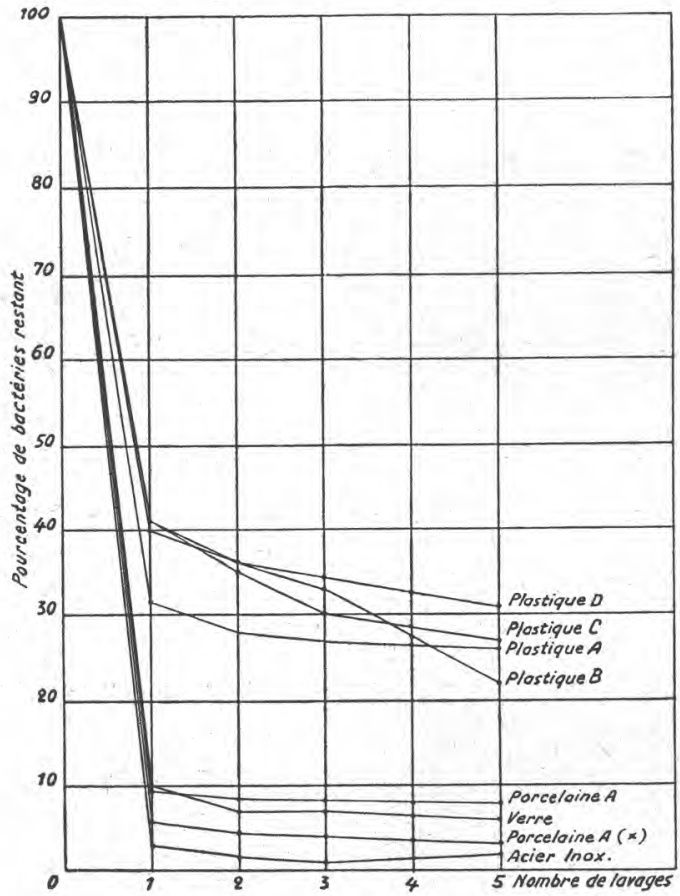

Figure 2

Pourcentage de M. aureus subsistant sur diverses surfaces endommagées après un certain nombre de lavages avec une solution détergente suivis de rinçage à $70^{\circ} \mathrm{C}$. ( dommages naturels.)

L'acier inoxydable conserve indéfiniment ses propriétés de résistance à la corrosion à condition que la surface soit propre après usage, ce qui implique l'élimination des résidus du lait et des dépôts calcaires. S'il arrive que des dépôts soient trop adhérents et qu'il soit impossible de les éliminer par rinçage ou lavage au détergent, il faut éviter à tout prix le grattage avec un outil métallique ordinaire ou un tampon en paille de fer. Il est conseillé plutôt d'utiliser une petite brosse en fil d'acier inoxydable 18/8. 
$2{ }^{\circ}$ Permanence des qualités de l'acier inoxydable au cours du temps et longévité des cuves, citernes et appareils.

L'acier inoxydable est encore un matériau idéal parce 'qu'il est très résistant à l'usure et aux chocs. Il n'est pas rare de rencontrer des installations datant de plus de vingt ans qui sont encore en excellent état et rendent les mêmes services qu'au premier jour. Il est par conséquent possible d'amortir les installations sur des périodes très longues.

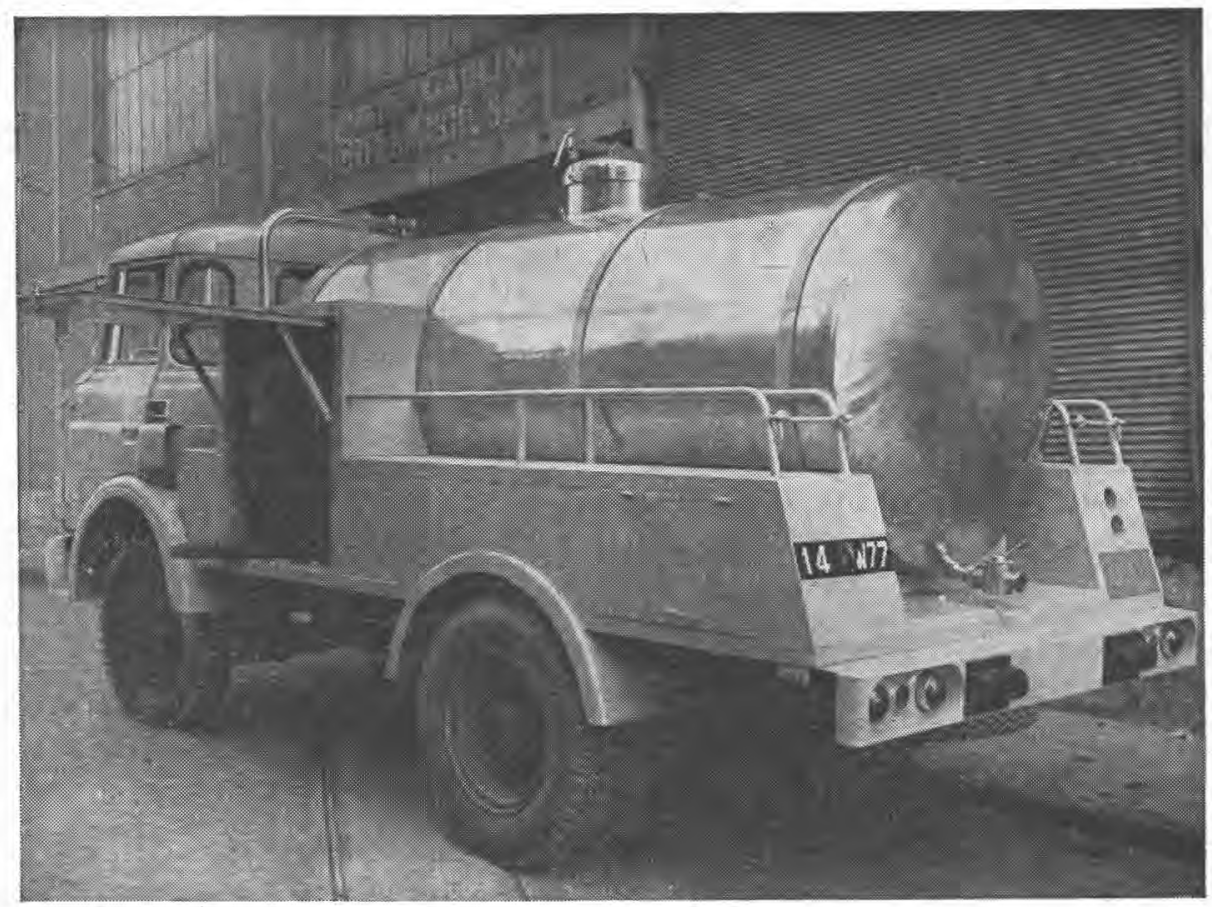

(Cliché aimablex ent communiqué par les Ets Breil et Martel)

Camion citerne pour le ramassage du lait

\section{IV. - Types d'acier inoxydable}

Aux U.S.A. des spécifications très strictes préconisent l'emploi des aciers chrome-nickel type 18/8 ou des aciers chrome-nickelmanganèse type 18/5/8. En Grande-Bretagne, Inde et Australie, les aciers 18/8 sont également spécifiés pour la fabrication des citernes de ramassage et les cuves de stockage du lait en vrac. 
En ce qui concerne le fini des surfaces, les normes américaines spécifient un fini meulé au grain d'abrasif $n^{\circ}$ 150-180 (fini $n^{\circ} 4$ ). Quoiqu'il en soit, les travaux de O. W. KAUFMANN et al. (1960, $J$. Dairy Sci., 43, 28) et de Masurovsky et Jordan $(1958, J$. Dairy Sci., 41, 1342) ont prouvé que l'efficacité des traitements de nettoyage et de stérilisation des aciers inoxydables 18/8 est identique quel que soit le fini de la surface (fini $2 B, 3,4,7$ ). En général, on accorde une préférence au fini no 4 pour des raisons purement esthétiques.

\title{
GONGLUSION
}

L'emploi de l'acier inoxydable s'étant développé dans bien d'autres réalisations, et ses productions s'étant par ailleurs nettement améliorées, ce matériau est aujourd'hui beaucoup plus abordable et d'un prix compétitif. C'est pourquoi ce nouveau mode de ramassage devrait pouvoir être entièrement réalisé dans ce matériel sans pour cela être d'un prix plus élevé, et cependant d'un amortissement plus facile parce que de bien plus longue durée.

\section{L'ACTION DE LA PRÉSURE SUR LA CASÉINe (fin)}

\author{
par \\ Ch. ALAIS et P. JOLLÈS
}

\section{DISGUSSION}

\section{Le substrat de la présure dans la réaction primaire.}

Les résultats que nous avons obtenus soutiennent la conception de WAUGH et V. Hipper [21] selon laquelle l'attaque immédiate et rapide de la présure porte sur la caséine $x$, d'une manière spécifique. Cette réaction primaire se produit avec de faibles quantités d'enzyme et précède toujours la coagulation.

Ces résultats sont :

a) La formation d'une proportion élevée d'azote non protéique avec la caséine $\times$ (5 fois plus qu'avec la caséine entière);

b) L'identité de la composition en acides aminés des caséinoglycopeptides provenant de différentes caséines de vache : caséine entière, caséine $\alpha$ et caséine $x$;

c) L'identité des acides aminés C-terminaux dans ces caséinoglycopeptides ; 